\title{
HIGH ENERGY PHOTOPRODUCTION OF NEUTRAL RHO MESONS ON COMPLEX NUCLEI
}

\author{
J. G. Asbury,* U. Becker, William K. Bertram, ${ }^{*} \dagger$ P. Joos, M. Rohde, and A. J. S. Smith* \\ Deutsches Elektronen-Synchrotron, Hamburg, Germany
}

and

C. L. Jordan and Samuel C. C. Ting

Department of Physics, Columbia University, New York, New York (Received 25 July 1967)

In an experiment performed at the Deutsches Elektronen-Synchrotron (DESY) 6.2-GeV electron synchrotron we have studied the production, at forward angles, of $\pi^{+} \pi^{-}$pairs from the reaction

$$
\gamma+A \rightarrow A+\pi^{+}+\pi^{-} .
$$

Measurements were made for various target nuclei $A$ (Be, C, $\mathrm{Al}, \mathrm{Cu}, \mathrm{Ag}$, and $\mathrm{Pb})$, momenta of the $\pi^{+} \pi^{-}$system $p(2.8,3.04,3.5$, and 4.5 $\mathrm{GeV} / c)$, and peak incident bremsstrahlung energies $k_{\max }(4.35$ and $6.02 \mathrm{GeV})$.

The objects of the experiment were fourfold: first, to search for possible $0^{+}, 1^{-}, 2^{+}, \cdots$ resonances by measuring the pair invariantmass distribution in the region $0.35-1.2 \mathrm{GeV} /$ $c^{2}$; second, to study the mechanism by which $\rho^{0}$ mesons are produced at high energy and low momentum transfers, where diffraction seems to dominate ${ }^{1}$; third, to determine the $\rho^{0}-N$ cross section using the diffraction model; and fourth, to determine the mass and width of photoproduced $\rho^{0}$ mesons.

The $\pi^{+} \pi^{-}$pairs were detected by a doublearm magnetic spectrometer which has been described in a previous publication. ${ }^{2}$ Through scintillation-counter hodoscopes, the kinematics of each event was then defined within the following uncertainty: $\delta p / p= \pm 2 \%, \delta \theta / \theta= \pm 3 \%$, and $\delta m / m= \pm 2 \%$. The separation of $\pi^{+} \pi^{-}$pairs from the background of $e^{+} e^{-}$pairs was accomplished by four large-aperture threshold Cherenkov counters and two lead-Lucite shower counters; protons were rejected by time-offlight techniques.

In the kinematical region accepted by our spectrometer $\left(\theta_{0}=4^{\circ}-17.2^{\circ}, p_{0}=1.2-2.25 \mathrm{GeV} /\right.$

$c, k_{\max }=4.35$ or $6.02 \mathrm{GeV}$ ), our measurements show that, for $\pi^{+} \pi^{-}$pair masses from 0.350 to $1.20 \mathrm{GeV} / c^{2}$, Reaction (1) is dominated by $\rho^{0}$-meson production. Within the statistical accuracy, $5 \%$, no other $\pi^{+} \pi^{-}$enhancements were observed. Therefore, as described below, we have used Reaction (1) to study the differential $\rho^{0}$ production cross section and to gain information as to the production mechanism of $\rho^{0}$ mesons.

To study the mechanism by which $\rho^{0}$ mesons are produced at high energy and low momentum transfer, we compare the data with the predictions of the diffraction models of Berman and Drell, Drell and Trefil, and Ross and Stodolsky, ${ }^{3}$ in which the forward $\rho^{0}$ production cross section may be expressed (in the laboratory system) as

$$
d \sigma / d \Omega=C(A) p^{2} f^{\prime}(p) f_{T}\left(R, t, \sigma_{\rho N}\right),
$$

where $p$ is the momentum of the $\rho^{0}$ meson, $R$ $=r_{0} A^{1 / 3}$ is the nuclear radius, $\sigma_{\rho N}$ is the total $\rho^{0}$-nucleon absorption cross section, and $t$ is the square of the momentum transfer to the nucleus. $C(A)$ is a normalization constant. The function $f^{\prime}(p)$ is proportional (following Berman and Drell) to $p\left(p^{2}+m^{2}\right)^{-1 / 2} \sigma_{\pi N}{ }^{2}(p)$, where $\sigma_{\pi N}(p)$ is the total $\pi$-nucleon cross section.

The function $f_{T}$, describing the production and reabsorption of $\rho^{0}$ mesons by the target nucleus, is found as follows: The forward production amplitude $f\left(0^{\circ}\right)$ on each individual target nucleon is attenuated by a factor $\exp \left(-\frac{1}{2} \sigma_{\rho} N^{D x}\right)$ where $x=$ distance traveled by $\rho^{0}$ inside the nucleus after its production, and $D=$ nucleon density function, a constant inside a sphere of radius $R$, zero elsewhere. Then, integrating over the nuclear volume assuming coherent production, we obtain $g_{T}\left(R, t, \sigma_{\rho N}\right)$, the forward $\rho^{0}$ production amplitude for the whole nucleus. From this, we define

$$
f_{T}\left(R, t, \sigma_{\rho N}\right)=\left|\frac{g_{T}\left(R, t, \sigma_{\rho N}\right)}{g_{T}\left(R, 0, \sigma_{\rho N}\right)}\right|^{2} .
$$

From Eq. (2) and the explicit forms of functions $f^{\prime}(p)$ and $f_{T}$, one sees that the diffraction model predicts the dependence of $d \sigma / d \Omega$ upon the atomic number of the target nucleus $A$, the momentum transfer $t$, and the $\rho^{0}$ momen- 


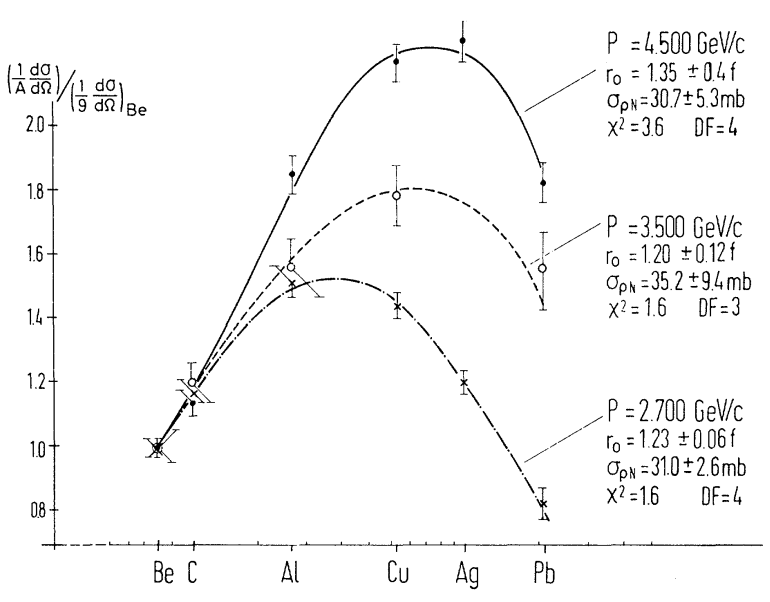

FIG. 1. Dependence of $A^{-1}(d \sigma / d \Omega)$ upon $A$, the atomic number of the target nucleus. Results are shown for average $\rho^{0}$ momentum $p=2.700,3.500$, and 4.500 $\mathrm{GeV} / c$. The curves are best fits to the model of Drell and Trefil as discussed in the text. The data are normalized to 1 for beryllium.

tum $p$, with two undetermined parameters $r_{0}$ and $\sigma_{\rho N}$.

By measuring $d \sigma / d \Omega$ as a function of one of $A, t$, or $p$, with the other two kept constant, one is able to determine $r_{0}$ and $\sigma_{\rho N}$, and to compare experiment with the diffraction-model predictions. The results of these comparisons are as follows:

(a) $A$ dependence. - The forward production cross sections $d \sigma / d \Omega$ were measured on targets of $\mathrm{Be}, \mathrm{C}, \mathrm{Al}, \mathrm{Cu}, \mathrm{Ag}$, and $\mathrm{Pb}$, all at the synchrotron energy of $6.0 \mathrm{GeV} / c$, fixed mass interval $720 \leqslant M \leqslant 820 \mathrm{MeV} / c^{2}$, and at three different spectrometer settings, $p=2.700,3.500$, and $4.500 \mathrm{GeV} / c$. The relative cross section normalized to $\mathrm{Be}$,

$$
\left(\frac{d \sigma}{d \Omega} / A\right) /\left(\frac{d \sigma}{d \Omega} / 9\right)_{\mathrm{Be}},
$$

is shown in Fig. 1. The cross section per nucleon increases coherently with increasing $A$ until $A \approx 64$; the yield then decreases with increasing $A$. The relative $A$-dependence behavior of the production cross section then yields information on the $\rho^{0}$-nucleon mean free path in nuclear matter. With the diffraction model of Drell and Trefil, ${ }^{3}$ the best fit of our data yields $r_{0}=1.29 \pm 0.04 \mathrm{~F}$, and $\sigma_{\rho N}=31.3 \pm 2.3$ $\mathrm{mb},{ }^{4}$ in good agreement with the total $\pi$-nucleon cross section $(\approx 30 \mathrm{mb})$ in the same energy region, as predicted by $\mathrm{SU}(6){ }^{5}$

(b) $t$ dependence. - The behavior of $d \sigma / d|t|$ as a function of four-momentum transfer $t$ $=(p-k)^{2}$ was obtained at a fixed $\theta_{0}=15^{\circ}$, a constant central $\rho^{0}$ laboratory momentum of 2.7 $\mathrm{GeV} / c$, and fixed mass interval $700 \mathrm{MeV} / c^{2}$ $<M<800 \mathrm{MeV} / c^{2}$. The momenta of $\pi^{+}\left(\pi^{-}\right)$could vary separately such that the total $|t|$ covered a region between $0.004(\mathrm{GeV} / c)^{2}$, and the production angle of the $\rho^{\circ}$ varied from $0^{\circ}$ to $6^{\circ}$. Data for $\mathrm{C}, \mathrm{Cu}$, and $\mathrm{Pb}$ targets $^{6}$ were taken and are shown in Figs. 2(a)-2(c).

The best fit of the quantity $d \sigma / d|t|$ by the form

$$
C_{1}(A) f_{T}(R, t, 31 \mathrm{mb})+C_{2}(A) e^{10 t}
$$

(where the second term represents the incoherent contribution from individual nucleons) yields a value for $r_{0}$ of $1.30 \pm 0.05 \mathrm{~F}$ in good agreement with that deduced above from the $A$ dependence. ${ }^{7}$ As shown in Fig. 2, for small momentum transfers $|t|<\left|t_{0}\right|$ (where $\left|t_{0}\right|^{1 / 2}=3.83 / R$ is the momentum transfer of the first diffraction minimum of a sphere of radius $R$ and constant opacity), the data are fitted well by (4). For $|t|$ $\ll\left|t_{0}\right|$ the $t$ dependence is given by

$$
(d \sigma / d|t|) \propto e^{a(A) t} .
$$

The best-fit values of $a(A)$ under these conditions are

$$
\begin{aligned}
a(\mathrm{C}) & =47 \pm 3(\mathrm{GeV} / c)^{-2} \\
a(\mathrm{Cu}) & =139 \pm 7(\mathrm{GeV} / c)^{-2} \\
a(\mathrm{~Pb}) & =290 \pm 12(\mathrm{GeV} / c)^{-2}
\end{aligned}
$$

Thus we see that $a(A)=a_{0} A^{2 / 3}$ with $a_{0}=10.5$ $\pm 1(\mathrm{GeV} / c)^{-2}$. The data exhibit a breaking point in the neighborhood of $t_{0}$. For momentum transfer $|t|>\left|t_{0}\right|$, the data fall off with a much smaller slope $e^{b t}$ with $b(\mathrm{Cu})=20 \pm 2(\mathrm{GeV} / c)^{-2}$ and $b(\mathrm{~Pb})=80 \pm 5(\mathrm{GeV} / c)^{-2}$. The data in this region are only in qualitative agreement with (4).

(c) $p$ dependence. - With knowledge that for $|t|<\left|t_{0}\right|, f_{T}\left(R, t, \sigma_{\rho N}\right)$ is in good agreement with the data, the behavior of $\left(f_{T} p^{2}\right)^{-1}(d \sigma / d \Omega)$ as a function of $p$ was studied for a fixed range of invariant mass $750 \mathrm{MeV} / c^{2}<M<780 \mathrm{MeV} / c^{2}$, over a momentum range from 2.5 to $4.5 \mathrm{BeV} /$ $c$. The data fall off slowly with $p$ in the same manner as the $\pi^{-}-p$ total cross section in this energy region. ${ }^{8}$ The comparison of $d \sigma / d \Omega$ with the forward $\pi-N$ diffraction cross section $(d \sigma /$ $d \Omega)_{\pi^{-} p}$ is shown in Fig. $2(\mathrm{~d})$, where one approximates the energy variation of the total $\pi^{-}-p$ cross section by an empirical formula 
(a)

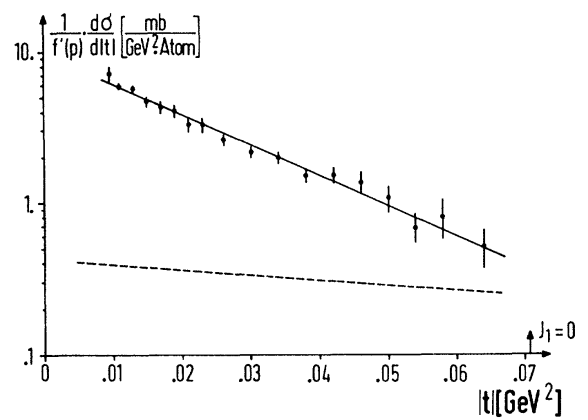

(b)

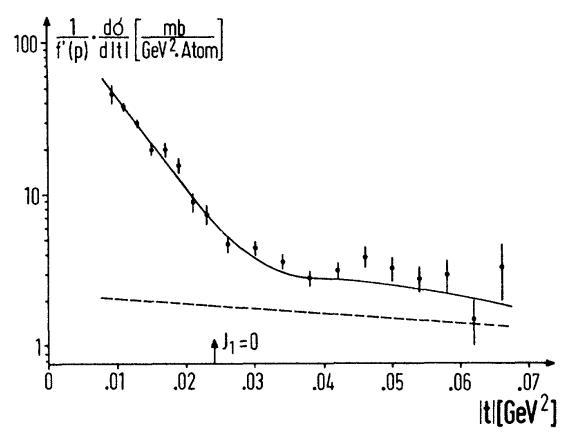

(c)

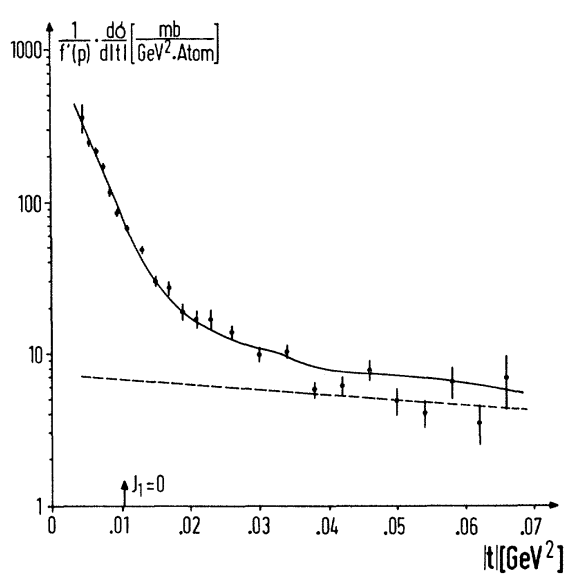

(d)

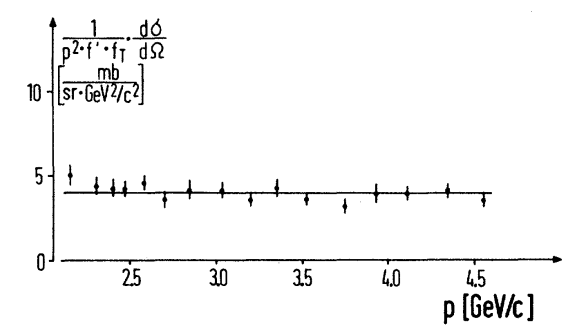

FIG. 2. (a)-(c) The quantity $f^{\prime}(p)^{-1}(d \sigma / d|t|)$ is shown as a function of $t$, the square of the momentum transfer to the nucleus, for carbon, copper, and lead targets, respectively. The curves are best fits to the model of Drell and Trefil with an additional incoherent contribution from individual nucleons. (d) Comparison of $d \sigma /$ $d \Omega\left(\gamma+A \rightarrow A+\rho^{0}\right)$ with $d \sigma(\pi N \rightarrow \pi N) / d \Omega$ as a function of $p$, for carbon. Only events having mass $m=m_{\rho} \pm 2 \%$ are included. The constancy of the ratio $\left[p^{2} f^{\prime}(p) f_{T}\right]^{-1}$ $\times(d \sigma / d \Omega)$ shows that the two cross sections have the same momentum dependence in the forward direction.
$1+(0.91 \mathrm{GeV} / c) / p$. Thus we define

$$
f^{\prime}(p)=\frac{[1+(0.91 / p)]^{2} p}{\left(p^{2}+m_{\rho}^{2}\right)^{1 / 2}}
$$

The quantity $\left[f^{\prime}(p) p^{2} f_{T}\right]^{-1} d \sigma / d \Omega$ is relatively independent of incident momentum $p$. This shows that $f_{T^{-1}} d \sigma / d \Omega$ varies as $f^{\prime}(p) p^{2}$ in agreement with the diffraction model of Berman and Drell.

From (a)-(c) we conclude that $\rho^{0}$ photo production in our energy region agrees with the predictions of diffraction production, and the cross section in the forward region can be described in the form

$$
d^{2} \sigma / d \Omega d m=C 2 m R(m) f_{T} f^{\prime}(p) p^{2},
$$

which for high energy and small momentum transfer becomes

$$
d^{2} \sigma / d \Omega d m=C 2 m R(m) p^{2} e^{a t} .
$$

To obtain $R(m)$ we grouped the events with respect to their invariant mass. The acceptance of each mass bin was determined by the Monte Carlo program, the dependence upon $p$ and $t$ of the production cross section being divided out.

Four mass spectra were obtained in this manner: carbon, $p=3.158 \mathrm{GeV} / c, k_{\max }=4.35 \mathrm{GeV} /$ $c$, and carbon, copper, lead, $p=4.500 \mathrm{GeV} / c$, $k_{\max }=6.00 \mathrm{GeV} / c$ (Fig. 3.) These were fitted by a function ${ }^{9,10}$

$$
U(m)=C 2 m R(m)+B(m)
$$

where

$$
R(m)=\left(\frac{m \rho}{m}\right)^{4} \frac{1}{\pi}\left(\frac{m \rho(m)}{\left(m_{\rho}{ }^{2}-m^{2}\right)^{2}+\Gamma^{2}(m) m_{\rho}^{2}}\right)
$$

with

$$
\Gamma(m)=\frac{m}{m_{\rho}}\left(\frac{\left(\frac{1}{2} m\right)^{2}-m_{\pi}^{2}}{\left(\frac{1}{2} m_{\rho}\right)^{2}-m_{\pi}^{2}}\right)^{3 / 2} \Gamma_{0} .
$$

The function $B(m)$ is a phenomenological background function expressed in terms of powers of $m$; the behavior of $B(m)$ was analyzed by fitting (6) to the four spectra above; because of coherency in $\rho^{0}$ production the background contribution to $U(m)$ decreases with increas ing $A$, and we found that the best-fit values of $m_{\rho}$ and $\Gamma_{0}$ are insensitive to the detailed form of $B(m)$. The best-fit values for lead are $\Gamma_{0}=130 \pm 5 \mathrm{MeV}$ and $m_{\rho}=765 \pm 5 \mathrm{MeV} / c^{2}$, 

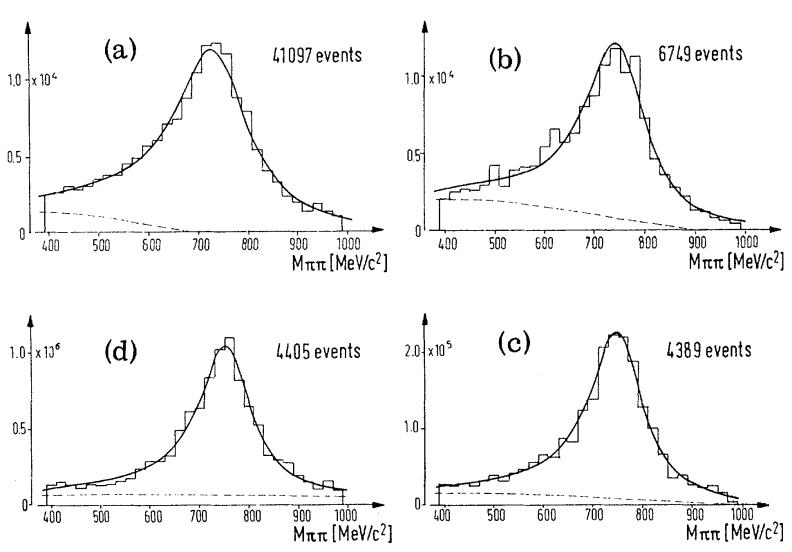

FIG. 3. Pion-pair invariant-mass spectra $\left[p^{2} f^{\prime}(p) f_{T}\right]^{-1}$ $\times(d \sigma / d \Omega d m)$ in units of $\mathrm{nb}(\mathrm{GeV} / c)^{-2}\left(\mathrm{MeV} / c^{2}\right)^{-1} \mathrm{sr}^{-1}$, for (a) carbon, $p=3.158 \mathrm{GeV} / c, k_{\max }=4.35 \mathrm{GeV} / c$; (b) carbon, $p=4.500 \mathrm{GeV} / c, k_{\max }=6.00 \mathrm{GeV} / c$; (c) copper, $p=4.500 \mathrm{GeV} / c, k_{\max }=6.00 \mathrm{GeV} / c$; and (d) lead, $p=4.500 \mathrm{GeV} / c$. The solid curve is the best fit of the data by the function $U(m)$ as described in the text. The dashed curve is the estimate of the nonresonant background contribution $B(m)$. As this background is small the best-fit values of $m_{\rho}$, the central mass of the $\rho^{0}$ meson, and $\Gamma_{0}$, its width, are insensitive to the explicit form of the background. The transverse polarization of the $\rho^{0}$ mesons was taken into account to obtain the absolute values shown.

which are in good agreement with the published $\pi-N$ results.

To determine the absolute forward $\rho^{0}$ production cross section in the laboratory system, the value of $C$ was obtained by normalizing (5) to the observed counting rate. The values of $C$ are for carbon,

$$
C=(3.72 \pm 0.23) \mathrm{mb} \mathrm{sr}^{-1}(\mathrm{GeV} / c)^{-2} \text {; }
$$

for copper,

$$
C=(64.0 \pm 6.4) \mathrm{mb} \mathrm{sr}^{-1}(\mathrm{GeV} / c)^{-2} ;
$$

for lead,

$$
C=(287.5 \pm 28.8) \mathrm{mb} \mathrm{sr}^{-1}(\mathrm{GeV} / c)^{-2} .
$$

Integrating (5) over $m$, one obtains, for $p$ $=4.5 \mathrm{GeV} / c, \theta=0.5^{\circ}$,

$$
\begin{gathered}
d \sigma / d \Omega(\mathrm{C})=5.0 \pm 0.4 \mathrm{mb} /(\mathrm{sr} \text { nucleon }), \\
d \sigma / d \Omega(\mathrm{Cu})=11.2 \pm 1.1 \mathrm{mb} /(\text { sr nucleon }), \\
d \sigma / d \Omega(\mathrm{Pb})=10.0 \pm 1.0 \mathrm{mb} /(\text { sr nucleon }) .
\end{gathered}
$$

The values of carbon and copper are in good agreement with results from Cambridge Electron Accelerator of $5.17 \pm 0.07$ and $10.5 \pm 0.2$ $\mathrm{mb} /\left(\mathrm{sr}\right.$ nucleon), respectively. ${ }^{1}$

In summary, the dependence upon $p, t$, and $A$ of the high-energy, small-angle $\rho^{0}$ photo- production cross section is in excellent agreement with the general features of the diffraction-dissociation model, and the mass and width of the photoproduced $\rho^{0}$ are consistent with the data from other reactions.

We are grateful for the support of Professor W. Jentschke and Professor P. Stähelin of DESY and Professor L. M. Lederman and Professor S. Devons of Columbia, who made this collaboration possible. We acknowledge many interesting discussions with Professor D. Frisch, Professor H. Joos, and Professor G. Weber; and we also thank Dr. H. O. Wüster and Dr. D. Lublow for extending to us the DESY computing facilities and $\mathrm{Mr}$. H. Kumpfert and the synchrotron staff for their skillful operation of the machine.

\footnotetext{
*Volkswagen Foundation Fellow at Deutsches Elektronen-Synchrotron.
}

$\dagger$ Present address: Physics Department, University of Illinois, Urbana, Illinois.

\$A Deutsches Elektronen-Synchrotron-Columbia collaboration, this work was supported in part by the U. S. Atomic Energy Commission.

${ }^{1}$ For earlier work on this subject, see L. J. Lanzerotti, R. B. Blumenthal, D. C. Ehn, W. L. Faissler, P. M. Joseph, F. M. Pipkin, J. K. Randolph, J. J. Russell, D. G. Stairs, and J. Tenenbaum, Phys. Rev. Letters 15, 210A (1965); Aachen-Berlin-Bonn-Hamburg-HeidelbergMünchen Collaboration, Nuovo Cimento 41A, 270 (1966).

${ }^{2}$ J. G. Asbury, W. K. Bertram, U. Becker, P. Joos, M. Rohde, A. J. S. Smith, S. Friedlander, C. Jordan, and Samuel C. C. Ting, Phys. Rev. Letters $\underline{18}, 65$ (1967).

${ }^{3}$ S. D. Drell and J. S. Trefil, Phys. Rev. Letters $\underline{16}$, 552, 832 (E) (1966); S. M. Berman and S. D. Drell, Phys. Rev. 133, B791 (1964); M. Ross and L. Stodolsky, Phys. Rev. $\underline{149}, 1172$ (1966).

${ }^{4} \mathrm{~A}$ similar result for $\sigma_{\rho N}$ was obtained from a strongabsorption optical model analysis of bubble-chamber data; Y. Eisenberg, G. E. Ronat, A. Brandstetter, A. Levy, and E. Gotsman, Phys. Letters 22, 217 (1966).

${ }^{5} \mathrm{H}$. Harari, private communication to S. D. Drell.

${ }^{6} \mathrm{For}$ the lead curve, the four points of lowest $|t|$ were obtained with $p=4.500 \mathrm{GeV} / c$ and were combined with the $p=2.700-\mathrm{GeV} / c$ data as described in Sec. (c).

${ }^{7}$ For $p$-nucleus scattering at high energy, see G. Bellettini, G. Cocconi, A. N. Diddens, E. Lillethun, G. Matthiae, J. P. Scanlon, and A. M. Wetherell, Nucl. Phys. 79, 609 (1966). For an excellent review on highenergy $\pi-p$ scattering, see M. N. Focacci and G. Giacomelli, CERN Report No. NP-66-18 (unpublished).

${ }^{8} \mathrm{~A}$. Citron et al., Phys. Rev. Letters $\underline{13}, 205$ (1964).

${ }^{9}$ J. D. Jackson, Nuovo Cimento 34,1644 (1964).

${ }^{10}$ For the use of $\left(m_{\rho} / m\right)^{4}$ factor in the Breit-Wigner formula, see M. Ross and L. Stodolsky, Phys. Rev. 149,1172 (1966); L. Stodolsky, Brookhaven National Laboratory Report No. BNL-11373, 1967 (unpublished). 